\title{
Anti-diabetic effects of pomegranate extracts in long-term high fructose-fat fed rats
}

\author{
Zahra Amri ${ }^{1 *}$ D, Mohamed Raâfet Ben Khedher ${ }^{1}$, Mohamed Sghaier Zaibi ${ }^{3}$, Wafa Kharroubi ${ }^{1}$, Mouna Turki ${ }^{2}$, \\ Fatma Ayadi ${ }^{2}$ and Mohamed Hammami ${ }^{1}$
}

\begin{abstract}
Background: A high-sugar or/and high-fat diets is a major risk factors for obesity. However, increased fruit and vegetable intake has been recently linked to obesity prevention. The aim of the present study was to investigate the preventive effects of pomegranate leaves (PL), juice (PJ) and peel (PP) extracts, on insulin resistance and oxidative stress in high fat and high fructose diet-induced obese rats.
\end{abstract}

Methods: Obesity in rats was induced by consumption of diet high in saturated fat and fructose (HFD) for a long period (12 weeks).

Results: Compared to the control group (CG) fed chow died, the high fat and high fructose diet (HFD) group showed a significant increase in the fasted plasma levels of glucose (29.8\%), insulin (45\%), amylase (70\%) and lipase (54\%). Moreover, HFD feeding has increased lipid peroxidation and protein carbonylation and decreased antioxidant enzymes levels. However, PL, PJ and PP treatment markedly prevents glucose intolerance, insulin resistance and oxidative stress and decrease amylase and lipase levels.

Conclusion: These findings highlight that a long-term intake of pomegranate extracts might be a potential alternative strategy for the prevention of a HFD induced insulin resistance and oxidative stress.

Keywords: Pomegranate parts, A high fructose-fat diet, Insulin resistance, Oxidative stress, Amylase, Lipase

\section{Background}

Obesity is a risk factor for several metabolic diseases, particularly type 2 diabetes $[1,2]$. Most patients with type 2 diabetes are overweight or obese, and the dramatic increase in the prevalence of type 2 diabetes was mostly related to an excessive body weight gain [3]. In fact, obesity is a condition marked by an expansion in subcutaneous and /or visceral adipose tissue. This fat accumulation results in an increase in a chronic systemic inflammation. This long term low grade inflammation induces a dysregulation in glucose and fatty acid metabolism, leading to insulin resistance $[4,5]$. Several previous studies, have

\footnotetext{
* Correspondence: zahraamri23@yahoo.fr

${ }^{1}$ Biochemistry Laboratory, LR12ES05 "Nutrition- Functional Foods and

Vascular Health", Faculty of Medicine, University of Monastir, Monastir, Tunisia Full list of author information is available at the end of the article
}

reported that HFD consumption enhances the accumulation of reactive oxygen species in liver resulting in chronic inflammation $[1,6,7]$ leading to insulin resistance and metabolic syndrome. Therefore, decreasing of HFD consumption can reduce liver inflammation and that may be a convenient strategy for preventing obesity-associated metabolic syndromes [6]. Recent epidemiological studies provided evidence linking dietary daily rations to incidence of obesity. Indeed, it has been reported that the increased consumption of high-sugar diets especially fructose-enriched diet $[8,9]$ or of high fat $[10,11]$ generated metabolic syndrome, rapidly promotes lipogenesis and worsens insulin resistance [12].

Currently there is a growing popularity of herbal therapies over conventional drugs, $40 \%$ of the compounds used in the pharmaceutical industry are derived from 
medicinal plants [13]. Moreover, the World Health Organization (WHO) announced that $80 \%$ of people in developing countries frequently use medicinal plants for their primary health care for various diseases. A wide variety of medicinal plants have been found to have a healthful effects in the treatment and the prevention of metabolic syndromes like diabetes and obesity [14-16]. In this context, much more attention has been focused particularly in fruit and vegetables consumption which was generally associated with lower prevalence of metabolic syndrome, like grape [9], green tea [17] and avocado oil [18].

The pomegranate, Punica granatum L. belongs to the punicaceae family and is cultivated in several countries, especially in the Mediterranean region. The juice, the seed oil, and the flower extracts have been described to have several beneficial effects in vitro as well as in vivo, such as antidiabetic, anti-obesity, anti-inflammatory, antioxidant, and antitumor effects [19]. They were highly rich in natural antioxidants such as flavonoids [20] and polyphenols [21] compared with any other fruit. In a previous study on the beneficial effects of pomegranate, Harzallah et al., [7] investigated the effect of three extracts (flowers, seed oil and peel) in C57bl/6 male mice, fed high fat and high fructose diet for 28 days. The authors observed that only pomegranate seed oil improved insulin sensitivity following the 4 weeks of treatment. Pomegranate flower and peel extracts have no effect on glucose homeostasis and insulin sensitivity, however they have exhibited a significant anti- inflammatory effect by decreasing the plasma levels of the pro-inflammatory cytokines IL- 6 and TNF- $\alpha$, and increasing the antiinflammatory cytokine IL-10. Taking these findings together, pomegranate extract particularly peel extract might have a beneficial effect on insulin sensitivity and glucose tolerance if it has been used for a longer period (over 6 weeks). Thus, the aim of our study is to investigate the effect of long term treatment (12 weeks) with pomegranate leaves (PL), juice (PJ) and peel (PP) extracts in male Wistar rats fed high fructose-high fat diet, on the glucose tolerance, the insulin sensitivity, the hepatic markers of oxidative stress, and on the activity of $\alpha$ amylase and lipase.

\section{Materials and methods Plant material}

Pomegranate leaves and fruits were reaped from Tounsi trees in Mahdia region, Tunisia. Authenticity of Tounsi cultivar was identified by Dr. Faten Zaouay, a taxonomist from the Higher Agronomic Institute (University of Sousse, Tunisia) and a voucher specimen was deposited in herbarium at the Faculty of Pharmacy (University of Monastir, Tunisia). Pomegranate juice and methanolic extracts of pomegranate leaves and peel were prepared as described in our previous study [22].

\section{Lipid extraction and analysis of fatty acid methyl esters (FAMEs)}

Protocols of lipid extraction and FAMEs analysis were carried out according to the protocol as described previously by our team [23].

\section{Experimental design}

Male Wistar rats (200-250 g) were obtained from the central pharmacy of Tunisia. For acclimatization conditions and maintenance requirements of rats, standard diet and HFD compositions, show data in our previous study [22]. Five groups (6 animals in each group) were divided. One group was fed with a standard diet only (control group CG). One group was fed with HFD only (obese control group HFD). The three remaining groups were fed with HFD and received daily per gavage one of three pomegranate extracts at a dose of $250 \mathrm{mg} / \mathrm{kg}$ of BW, pomegranate juice (Group HFD+ PJ) pomegranate peel (group HFD+ PP) and pomegranate leaves (group HFD+ PL). Throughout the study, body weight and food intake were monitored respectively twice a week and daily. At the end of the experiment, and after an overnight fast the rats were sacrificed by decapitation and the blood was taken for biochemical analysis.

\section{Insulin tolerance test (ITT) and Oral glucose tolerance test (OGTT)}

ITT and OGTT were carried out respectively at 10th and 11th week of treatment with pomegranate extracts. For the ITT, the overnight fasted rats received $0.75 \mathrm{IU} /$ $\mathrm{kg}$ of insulin solution by intraperitoneal injection, and blood glucose was measured at $0,30,60$ and 90 min following the injection.

For the OGTT, $2.5 \mathrm{~g} / 10 \mathrm{ml} / \mathrm{kg}$ of glucose dissolved in water were loaded to overnight fasted rats by oral gavage and blood samples were collected by incision from the tail for glucose measurement at $0 \mathrm{~min}$ before, and, 30, 60, 90 and $120 \mathrm{~min}$ after the glucose load. Glucose was determined immediately by a portable glucometer.

\section{Biochemical analysis}

The levels of plasma glucose were measured using the glucose oxidase-PAP kit (Biomaghreb, Tunis City, Tunisia). The levels of plasma insulin were measured using RayBio Mouse Insulin ELISA kit (RayBiotech. Catalog \#: ELM-Insulin). The $\alpha$-amylase activity was evaluated according to kinetic method using a commercial kit (BIOLABO ref. 80,023, Maizy, France). The lipase activity was evaluated also according to kinetic method using a commercial kit (BIOLABO ref. 99,891, Maizy, France). 


\section{Hepatic markers of oxidative stress}

Liver malondialdehyde (MDA) as a marker of lipid peroxidation was measured according to reference [24]. Protein carbonylation (PC) as a marker of protein oxidative damage was quantified according to reference [25]. Superoxide dismutase (SOD) activity was carried out following the method cited in reference [26]. Glutathione peroxidase (GPx) activity was evaluated as described by reference [27].

\section{Statistical analysis}

Statistical analysis was performed using SPSS version 21. Duncan's test was used to analyze the data and the criterion for statistical significance was $p<0.05$.

\section{Results}

Fatty acid composition

The fatty acid composition of the studied pomegranate extracts are shown in Table 1. Results showed 33 identified fatty acids ranging from C12:0 to very long chain fatty acids such as C24:0. Unsaturated fatty acids (UFA) were the main fraction in the all extracts and it contained MUFA, Di-UFA and Tri-UFA. Furthermore, four different families of unsaturated fatty acids (UFAs) were detected in our samples including omega $3(w-3)$, omega 6 (w-6), omega 7 (w-7) and omega 9 (w-9) fatty acids. Polyunsaturated fatty acids (PUFAs) are reported very important for human health. They prevent and reverse high-fat-diet induced adipose tissue inflammation and insulin resistance [28, 29], decrease blood cholesterol levels, prevent CVD mortality [30] and inhibit apoptosis and neuroinflammation [31]. PJ was more concentrated in UFA and PL presented the highest percentage of SFA. In generally no significative difference was shown in SFA/UFA ratio. The dominant UFA are C18:1 w9 (cis) for PP, C18:1 w7 (cis) for PJ and C18:2 (t9, c12) for PL. The major SFA are C16:0 and C17:0. Unlike to pomegranate seeds oils, these pomegranate extracts contained fewer amounts of Conjugated Linolenic acids (Clna) especially punicic acid C18:3 (c9, t11, c13) which was reported by several researchers as the predominant FA in pomegranate seed oil and its content can exceed $70 \%$. The trans/cis ratio seems very low in generally. Researchers reported that the low trans/cis ratio has health benefits. In fact, high trans fatty acids intake affect serum lipid levels and increased coronary heart disease mortality, and cardiovascular disease (CVD) incidence [32].

\section{Effect on glucose homeostasis and insulin sensitivity}

When compared to the group fed chow diet, the fasted levels of plasma glucose and insulin were increased respectively by $30 \%$ and $44 \%$ in HFD group after the 12 weeks of High fat and high fructose diet feeding (Table.2). The treatment with pomegranate juice leaves
Table 1 Fatty acid composition of individual lipid classes of pomegranate seeds

\begin{tabular}{|c|c|c|c|}
\hline FATTY ACIDS & $\mathrm{PL}$ & PJ & PP \\
\hline $\mathrm{C} 12: 0$ & $0,49 \pm 0,13$ & $2,28 \pm 0,27$ & $0,74 \pm 0,08$ \\
\hline C14:0 & $0,71 \pm 0,06$ & $5,24 \pm 1,13$ & $1,61 \pm 0,41$ \\
\hline C16:0 & $20,71 \pm 1,64$ & $14,63 \pm 0,64$ & $16,01 \pm 1,1$ \\
\hline $\mathrm{C} 17: 0$ & $16,75 \pm 0,12$ & $12,90 \pm 2,5$ & $18,77 \pm 2,06$ \\
\hline C18:0 & $4,76 \pm 0,27$ & $2,96 \pm 0,07$ & $3,96 \pm 0,12$ \\
\hline C20:0 & $0,39 \pm 0,06$ & $0,17 \pm 0,06$ & $0,82 \pm 0,13$ \\
\hline C22:0 & $0,20 \pm 0,02$ & $0,21 \pm 0,05$ & $0,63 \pm 0,08$ \\
\hline C24:0 & $0,16 \pm 0,01$ & $0,84 \pm 0,11$ & $0,21 \pm 0,01$ \\
\hline C14:1 w9 & $3,48 \pm 0,75$ & $0,96 \pm 0,12$ & $1,77 \pm 0,06$ \\
\hline C16:1 w7 (trans) & $1,55 \pm 0,008$ & $0,33 \pm 0,04$ & $1,06 \pm 0,004$ \\
\hline C16:1 w7 (cis) & $0,67 \pm 0,08$ & $0,19 \pm 0,04$ & $0,57 \pm 0,04$ \\
\hline C17:1w8 & $0,58 \pm 0,15$ & $0,25 \pm 0,01$ & $0,24 \pm 0,12$ \\
\hline C18:1 w9 (cis) & $7,57 \pm 0,11$ & $10,93 \pm 0,5$ & $18,04 \pm 2,51$ \\
\hline C18:1 w9 (trans) & $9,8 \pm 0,5$ & $0,14 \pm 0,03$ & $8,54 \pm 0,19$ \\
\hline C18:1 w7(cis) & $0,74 \pm 0$ & $31,60 \pm 0,1$ & $0,26 \pm 0,13$ \\
\hline C2O:1 w9 & $0,36 \pm 0,15$ & $0,36 \pm 0,11$ & $0,3 \pm 0,15$ \\
\hline C24:1 w9 & $0,2 \pm 0,07$ & $0,21 \pm 0,07$ & $0,04 \pm 0,01$ \\
\hline $\mathrm{C} 18: 2(\mathrm{t} 9, \mathrm{c} 12)$ & $19 \pm 1,39$ & $8,42 \pm 0,06$ & $11,67 \pm 0,12$ \\
\hline C18:2 (c9,t12) & $0,62 \pm 0,2$ & $1,42 \pm 0,4$ & $1,70 \pm 0,05$ \\
\hline C18:2 w6 (c9,c12) & $0,55 \pm 0,13$ & $0,24 \pm 0,13$ & $0,33 \pm 0,07$ \\
\hline C18:3 w6 & $0,31 \pm 0,09$ & $1,56 \pm 0,11$ & $0,72 \pm 0,32$ \\
\hline C18:3 w3 (cis) & $1,63 \pm 0,12$ & $1,42 \pm 0,05$ & $1,69 \pm 0,09$ \\
\hline C18:2 (t9,t11) & $1,96 \pm 0,11$ & $0,16 \pm 0,03$ & $0,62 \pm 0,04$ \\
\hline $\mathrm{C} 18: 2(\mathrm{c} 11, \mathrm{t} 13)$ & $0,88 \pm 0,73$ & $0,09 \pm 0,01$ & $2,4 \pm 0,15$ \\
\hline C18:2 (t10,c12) & $0,04 \pm 00$ & $0,3 \pm 0,06$ & $0,96 \pm 0,01$ \\
\hline $\mathrm{C} 2 \mathrm{O}: 2$ & $0,28 \pm 0,09$ & $0,14 \pm 0,002$ & $0,25 \pm 0,005$ \\
\hline C2O:3 w6 & $0,64 \pm 0,03$ & $0,04 \pm 0,01$ & $0,45 \pm 0,08$ \\
\hline $\mathrm{C} 2 \mathrm{O}: 3 \mathrm{w} 3$ & $0,93 \pm 0,05$ & $0,36 \pm 0,05$ & $0,68 \pm 0,1$ \\
\hline C18:3 (c9,t11,c13) & $0,16 \pm 0,02$ & $0,08 \pm 0,008$ & $1,23 \pm 1,5$ \\
\hline $\mathrm{C} 18: 3(\mathrm{c} 8, \mathrm{t} 10, \mathrm{c} 12)$ & $2 \pm 0,16$ & $0,05 \pm 0,02$ & $0,9 \pm 0,18$ \\
\hline $\mathrm{C} 18: 3(\mathrm{c} 9, \mathrm{t} 11, \mathrm{t} 13)$ & $1,43 \pm 0,36$ & $1,52 \pm 0,01$ & $1,67 \pm 0,12$ \\
\hline $\mathrm{C} 18: 3(\mathrm{t} 9, \mathrm{t} 11, \mathrm{c} 13)$ & $0.36 \pm 0,02$ & $0,41 \pm 0,03$ & $0,47 \pm 0,10$ \\
\hline $\mathrm{C} 18: 3(\mathrm{t} 9, \mathrm{t} 11, \mathrm{t} 13)$ & $0,53 \pm 0,11$ & $0,035 \pm 0,01$ & $0,39 \pm 0,03$ \\
\hline ¿SFA & $44,19 \pm 1.73^{\mathrm{a}}$ & $39,26 \pm 2^{b}$ & $42,8 \pm 1.6^{\mathrm{a}}$ \\
\hline$\sum$ PUFA & $56,04 \pm 1.9$ & $60,32 \pm 1,63$ & $57,06 \pm 3.5$ \\
\hline UFA/SFA ratio & $1,26 \pm 0,05$ & $1,3 \pm 0,07$ & $1.1 \pm 0.12$ \\
\hline trans/cis UFA ratio & $0.77 \pm 0.04^{\mathrm{a}}$ & $0.015 \pm 0.002^{c}$ & $0.4 \pm 0.04^{b}$ \\
\hline
\end{tabular}

Each value represents the mean of three determinations $(n=3) \pm$ SD. Means with different letters within the same row column were significantly different at the level of $p<0.05$

and peel resulted in a significant reduction in fasted plasma glucose and insulin levels. Consequently, the homeostatic index of insulin resistance (HOMA-IR) which is used to quantify insulin resistance [33, 34], was 
Table 2 Effect of HFD and pomegranate extracts administration plasma glucose, insulin levels and index (HOMA-IR)

\begin{tabular}{llllll}
\hline & CG & $H F D$ & $H F D+P J$ & $H F D+P L$ & $H F D+P P$ \\
\hline Glucose $(\mathrm{mg} / \mathrm{dl})$ & $90.5 \pm 9.02^{* *}$ & $129 \pm 6.32$ & $104.83 \pm 9.17^{* *}$ & $114 \pm 9.40^{*}$ & $113.66 \pm 14.26^{*}$ \\
Insulin $(\mu \mathrm{l} / \mathrm{U} / \mathrm{ml})$ & $30 \pm 11.06^{* *}$ & $54.60 \pm 8.32$ & $33 \pm 6.84^{* *}$ & $31 \pm 6.93^{* *}$ & $37 \pm 7.14^{*}$ \\
Insulin sensitivity index (HOMA-IR) & $2600.5 \pm 883.9^{* *}$ & $7013.03 \pm 1306.8$ & $3459.20 \pm 518.59^{* *}$ & $3494.9 \pm 697.9^{* *}$ & $4270 \pm 670.7^{* *}$
\end{tabular}

Results are shown as the mean \pm SEM $(n=6) .{ }^{*} p<0.05 ;{ }^{* *} p<0.01$ versus HFD group, HFD High Fructose-fat Diet, pomegranate leaves $(P L)$, pomegranate juice $(P J)$ and pomegranate peel $(P P)$

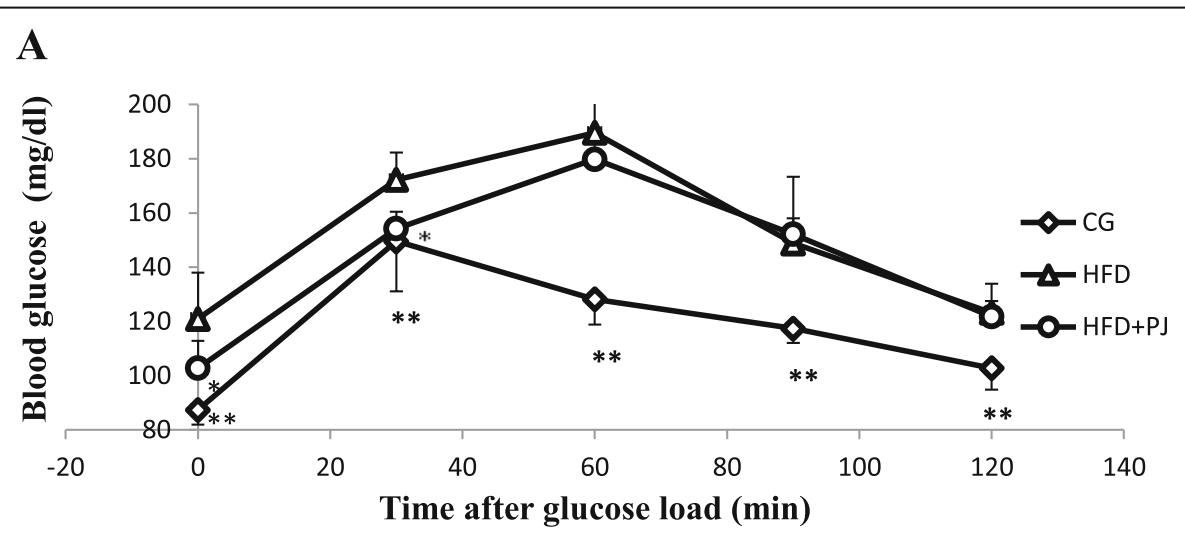

$\mathbf{B}$

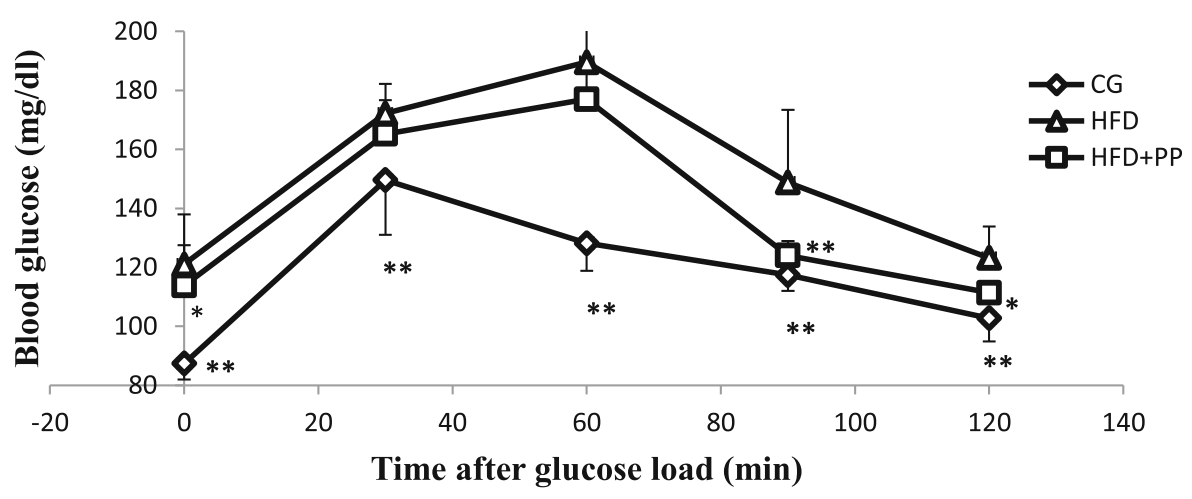

C

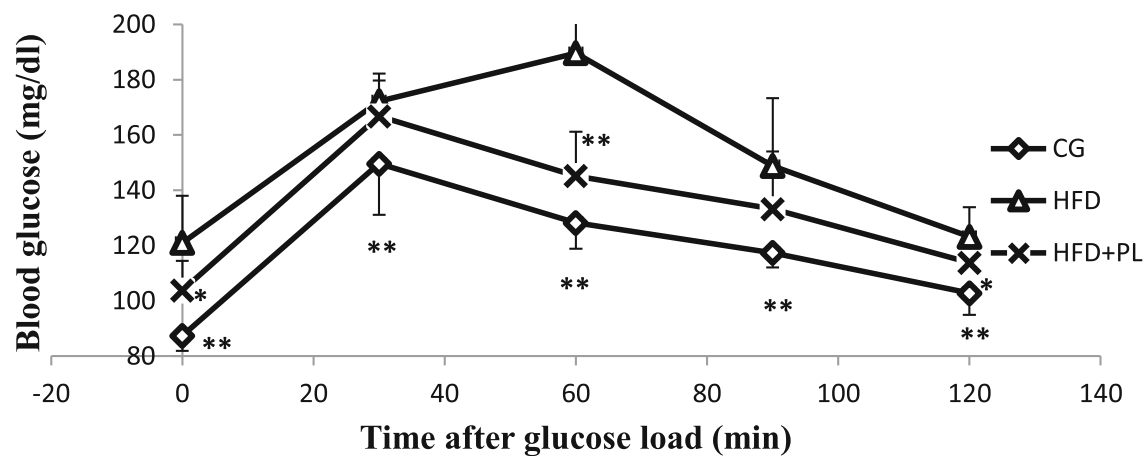

Fig. 1 Effect of pomegranate extracts supplementation on glucose tolerance curve in rats during 11 weeks experimental period. Results are shown as the mean \pm SE $(n=6) .{ }^{*} p<0.05 ;{ }^{* *} p<0.01$ versus HFD group, pomegranate juice $(\mathbf{a})$, pomegranate peel $(\mathbf{b})$ and pomegranate leaves (c) 


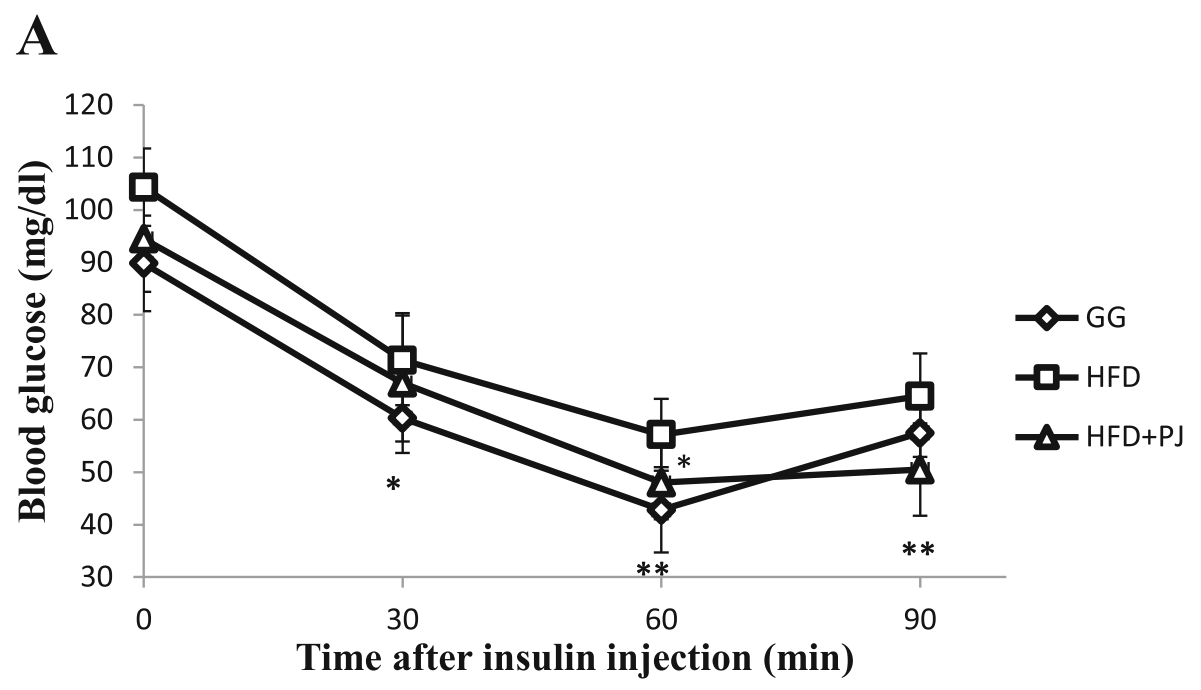

B

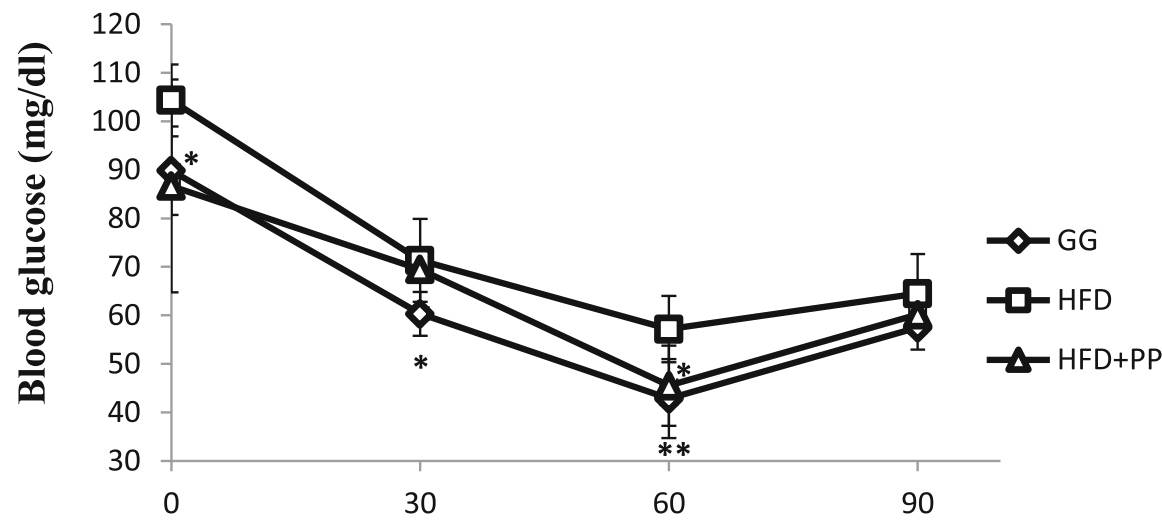

Time after insulin injection (min)

C

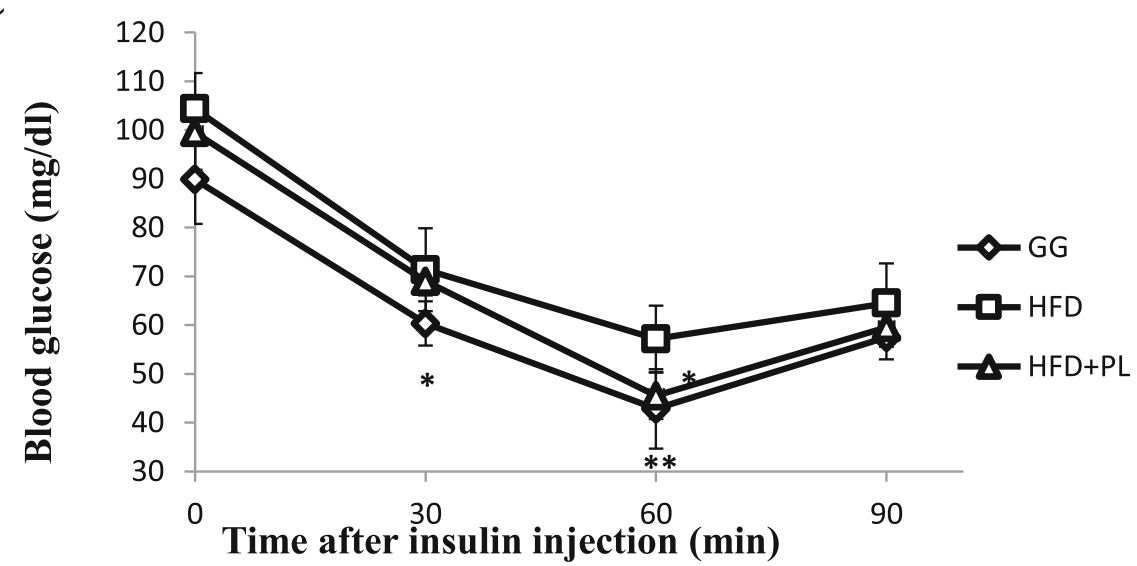

Fig. 2 Effect of pomegranate extracts supplementation on the insulin tolerance curve in rats during 11 weeks experimental period. Results are shown as the mean \pm SE $(n=6) .{ }^{*} p<0.05 ;{ }^{* *} p<0.01$ versus HFD group, pomegranate juice (a), pomegranate peel (b) and pomegranate leaves $(\mathbf{c})$ 

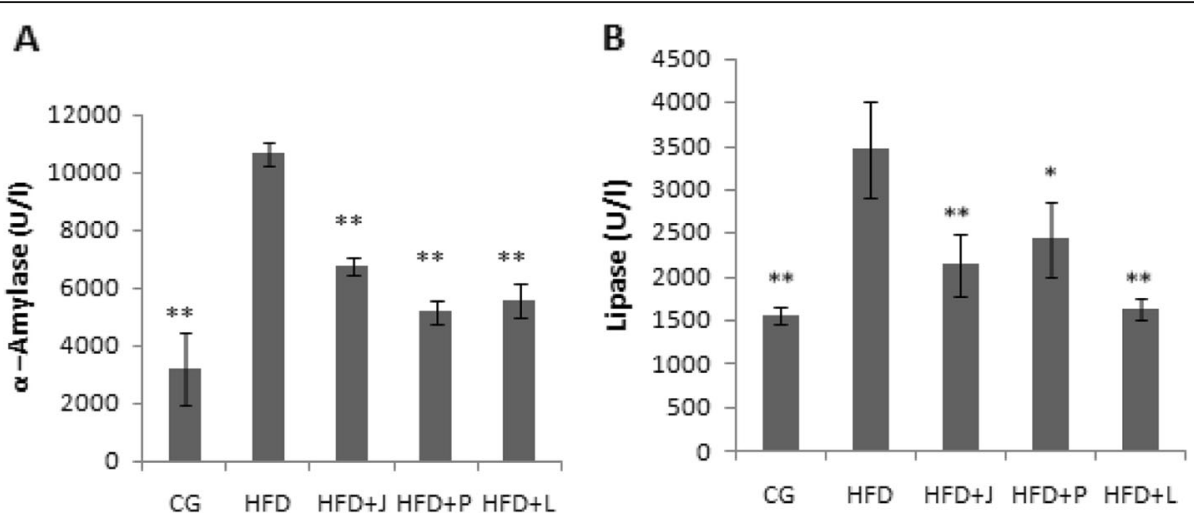

Fig. 3 Effect of pomegranate extracts administration on serum amylase (a) and lipase (b) levels. Results are shown as the mean $\pm \operatorname{SE}(n=6)$. ${ }^{*} p<$ $0.05 ;{ }^{* *} p<0.01$ versus HFD group, pomegranate juice (PJ), pomegranate peel (PP) and pomegranate leaves (PL)

respectively reduced by half for juice and leaves extracts and by $39.1 \%$ for peel extract indicating a significant improvement in insulin sensitivity by the three pomegranate extracts.

To further evaluate the effect of pomegranate extracts on glucose metabolism and insulin sensitivity, we have performed an oral glucose and insulin tolerance tests. Both tests resulted in incremental changes in animal plasma glucose.

After animals received glucose solution, the incremental plasma glucose peaked at $60 \mathrm{~min}$. Results illustrated in Fig.1; show that HFD feeding impaired glucose tolerance in rat. In fact, HFD group had higher level of blood glucose than control group after 30, 60, 90 and 120 min after oral glucose gavage (Fig. 1). Pomegranate leaves-treated HFD rats had significantly lower levels of plasma glucose than HFD group at $60 \mathrm{~min}$ after oral glucose load $(p<$ 0.01 ) and the levels tended to remain low (Fig. 1c). Pomegranate peel-treated HFD rats showed also a slight decrease in plasma glucose at $60 \mathrm{~min}$ and a significant decrease after 90 and $120 \mathrm{~min}$ after oral glucose gavage compared with HFD group (Fig. 1b). Although, results in Fig. 1a demonstrated that HFD rats treated with pomegranate juice were glucose intolerant. In fact, there were no remarkable differences in the glucose level at any timepoint with HFD group after glucose administration.

During insulin tolerance test, results show that HFD feeding impaired insulin sensitivity in rat (Fig. 2). In fact, the plasma glucose concentrations were decreased significantly at 30 and 60 min for all HFD feeding groups then restored slightly at $90 \mathrm{~min}(p<0.05)$. The HFD group had higher level of blood glucose than control group after 30 and $60 \mathrm{~min}$ of insulin injection. All pomegranate extracts have preventive effect and decreased significantly the levels of plasma glucose at $60 \mathrm{~min}$ after insulin injection $(p<0.05)$ compared to HFD group. This indicates that pomegranate extracts consumption can significantly improve glucose homeostasis and insulin resistance.

\section{Effect on the plasma levels of amylase and lipase}

The effect of pomegranate extracts treatment on $\alpha$ amylase and lipase activities are reported respectively in Fig. 3a and b. The high fat and high fructose feeding induced a significant increase in the serum $\alpha$-amylase activity by $70 \%$ when compared to control rats $(p<0.001)$. Interestingly, the oral treatment with PJ, PP and PL resulted in a significant decrease in $\alpha$-amylase activity by $30 \%, 50 \%$ and $49 \%$ respectively when compared to the HFD group.

Compared to the control group fed chow diet, the HFD feeding induced a potent increase $(p<0.01)$ in the plasma lipase activity. However, the long term treatment with PJ, PP and PL extracts reverted back the lipase activity in plasma by $37 \%, 51 \%$ and $28 \%$ respectively.

Table 3 Effect HFD and pomegranate extracts administration on hepatic oxidative markers

\begin{tabular}{llllll}
\hline & CG & $H F D$ & $H F D+P J$ & $H F D+P P$ & $H F D+P L$ \\
\hline GPX (U/g P) & $5.20 \pm 0.83^{* *}$ & $2.8 \pm 0.23$ & $3.4 \pm 0.7$ & $2.13 \pm 0.54$ & $4.04 \pm 0.91^{*}$ \\
SOD (U/mg P) & $8.24 \pm 0.45^{* *}$ & $3.62 \pm 0.56$ & $5.92 \pm 0.36^{* *}$ & $7.32 \pm 0.41^{* *}$ & $7.73 \pm 0.78^{* *}$ \\
MDA (nmol/mgP) & $0.29 \pm 0.02^{* *}$ & $0.5 \pm 0.07$ & $0.3 \pm 0.06^{*}$ & $0.25 \pm 0.07^{* *}$ & $0.30 \pm 01^{*}$ \\
PC ( $\mu$ M) & $13.67 \pm 1.27^{* *}$ & $18.51 \pm 2.63$ & $15.12 \pm 2.15^{*}$ & $12.43 \pm 1.72^{* *}$ & $14.42 \pm 0.48^{*}$ \\
\hline
\end{tabular}

Results are shown as the mean $\pm \operatorname{SEM}(n=6) .{ }^{*} p<0.05 ;{ }^{* *} p<0.01$ versus HFD group, HFD High Fructose-fat Diet, pomegranate leaves $(P L)$, pomegranate juice $(P J)$ and pomegranate peel $(P P)$ 


\section{Effect on hepatic oxidative markers}

Table 3 shows the beneficial effect of pomegranate extracts on hepatic oxidative stress status in HFD fed rats. Results revealed that HFD increased oxidative injury to lipids (by $+42 \%$ for MDA level) and proteins (by + $26.14 \%$ for PC level) and reduced significantly levels of SOD (by 56\%) and GPx (46\%) compared to control group. Whereas, the levels of MDA and PC were significantly decreased in all pomegranate extracts treated HFD groups. This indicates that pomegranate juice, peel and leaves alleviated lipid peroxidation and protein carbonylation. Furthermore, pomegranate extracts consumption improved significantly hepatic antioxidant enzymes levels such as SOD and GPx in treated groups compared to HFD group. PL exhibited a significant increase in GPx level, however the increased effects generated by PP and PL were statistically non-significant.

\section{Discussion}

Fructose, a dietary monosaccharide is known as a contributor to nearly all of the classic manifestations of the metabolic syndrome, including insulin resistance, hyperinsulinemia, hypertension, and dyslipidemia [35]. For that, several previous researches have used high fructose diet supplementation as an excellent animal model to study metabolic syndrome, type 2 and obesity-induced inflammation and insulin resistance, as well as to investigate the modulating effects of dietary components on progression of these diseases [36-38]. Within this framework, we utilized this model to investigate the potential beneficial effects of the long term treatment with pomegranate leaves, juice and peel extracts. Our results show that the consumption of HFD for 12 weeks markedly induces an increase in body weight (Show our previously published results), glycemia associated with insulinemia and, consequently, an insulin resistance. These findings are consistent with previous studies [36].

Body weight reduction shown in HFD rats may be explained by the consumption of energetic food rich in saturated fats (lard) and carbohydrate (fructose) which stimulates appetite by increasing ghrelin levels [39] and blocked satiety by decreasing leptin levels [40]. During OGTT and ITT, the incremental plasma glucose and insulin concentrations were significantly increased in HFD groups, indicating that HFD feeding markedly impaired insulin-stimulated glucose uptake in peripheral tissues. The action mechanism of fructose-fat feeding on insulin resistance remain incomprehensible but some explications have been proceeded including insulin signaling alteration [17], the enzymatic activities of carbohydrate metabolism [12] and excessive reactive oxygen species (ROS) production. In this study, we may explain fructose-induced hyperglycemia by increase of ROS and the decrease of the antioxidant protection system in various tissues like liver. In fact, our findings show that excessive fructose intake causes lipid and protein peroxidation and thus the inhibition of hepatic antioxidant enzyme like SOD and GPx. Others previous investigations reported similar results the same rats model [41, 42].

Results show that pomegranate leaves, peel or juice supplementation prevent body-weight gain (Show our previously published results [22]), hyperglycemia and hyperinsulinemia. Furthermore, they remarkably attenuate the impairment of insulin-stimulated glucose disposal in insulin resistant rats [9]. Moreover, pomegranate extracts protect hepatic antioxidant enzymes and decrease lipid and protein peroxidation. The preventing effect of pomegranate extracts can be related to the antioxidant properties of their active components such as polyphenols, flavonoids and anthocyanins [21, 43].

The inhibition of amylase activity by pomegranate extracts (which has been also reported by other authors $[44,45])$ resulted in a decrease in intestinal absorption of carbohydrates by suppressing their brakedown [46]. This reduction of the intestinal absorption of carbohydrates may explain the lowering effect of pomegranate extracts in fasted plasma glucose. Moreover, the decrease in intestinal carbohydrates absorption contributes to the reduction in energy intake leading to the weight loss observed in pomegranate extracts-treated HFD rats (Show our previously published results [22]).

The improvement of insulin sensitivity observed in pomegranate treated animals might be related to the antiinflammatory properties of the fruit extracts described by Harzallah et al. [7]. Indeed the decrease in proinflammatory cytokines such as TNF- $\alpha$ or IL- 6 may have a beneficial effect by improving glucose uptake in target tissues such as skeletal muscle and/or adipose tissue.

Moreover, the restoration of plasma lipids profile (Show our previously published results [22]) by pomegranate extracts supplementation may be attributed to the diminution of the fat and cholesterol absorption resulting from the inhibition of lipase, the key enzyme in lipid metabolism. Pomegranate extracts may contain active compounds considered as pancreatic lipase inhibitors [47]. Our findings are in line with a previous report. In fact, a screening of in vitro pancreatic lipase inhibition by extracts of fruits, vegetables, legumes and cereals, containing high levels of anthocyanin reported that pomegranate juice had the highest level of anthocyanin and was the best inhibitor of pancreatic lipase [48].

\section{Conclusion}

Our study indicates that the long-term treatment of high fat and high fructose fed rats with pomegranate leaves, peel or juice extracts, improves glucose tolerance, prevents insulin resistance and reduces carbohydrates and lipid absorption by decreasing $\alpha$-amylase and lipase activities. 


\section{Abbreviations}

HFD: High Fat High Fructose Diet; BW: Body Weight; CG: Control Group; PL: Pomegranate leaves; PJ: Pomegranate Juice; PP: Pomegranate Peel; MDA: Malondialdehyde; SOD: Superoxide Dismutase; GPx: Glutathione peroxidase; PC: Protein Carbonylation; ROS: Reactive Oxygen Species (ROS); ITT: Insulin Tolerance Test; OGTT: Oral Glucose Tolerance Test; HOMAIR: Homeostatic Index of Insulin Resistance

\section{Acknowledgements}

We thank the Ministry of High Education, scientific research and Technology of Tunisia for funding the study.

\section{Authors' contributions}

Z. Amri designed the study, participated in data collection, analysis, interpretation, and writeup. MR. Ben Kheder participated in study design, data collection and write-up. MS. Zaibi participated in study design and critically revised the manuscript. W. Kharroubi and M. Turki drafted the manuscript and critically revised the manuscript. A. Elfeki and F. Ayadi provided materials and reagents. M. Hammami critically revised the manuscript. All authors read and approved the final manuscript.

\section{Funding}

Not applicable.

\section{Availability of data and materials \\ Not applicable.}

\section{Ethics approval and consent to participate}

The study was approved by Animal Ethics Committee of the University of Sfax (Sfax, Tunisia) for the care and use of laboratory animals.

\section{Consent for publication}

Not applicable.

\section{Competing interests}

The authors declare that they have no competing interests.

\section{Author details}

'Biochemistry Laboratory, LR12ES05 "Nutrition- Functional Foods and Vascular Health", Faculty of Medicine, University of Monastir, Monastir, Tunisia. ${ }^{2}$ Biochemistry Laboratory, $\mathrm{CHU}$ H. Bourguiba, Sfax, Tunisia. ${ }^{3}$ Buckingham Institute for Translational Medicine, Clore Laboratory, University of Buckingham, Buckingham, UK.

\section{Received: 19 February 2020 Accepted: 6 August 2020}

Published online: 13 August 2020

\section{References}

1. Tuzcu Z, Orhan C, Sahin N, Juturu V, Sahin K. Cinnamon polyphenol extract inhibits Hyperlipidemia and inflammation by modulation of transcription factors in high-fat diet-fed rats. Oxidative Med Cell Longev. 2017;2017:10 https://www.hindawi.com/journals/omcl/2017/1583098/.

2. Lavie CJ, De Schutter A, Parto P, Jahangir E, Kokkinos P, Ortega FB, et al. Obesity and prevalence of cardiovascular diseases and prognosis-the obesity paradox updated. Prog Cardiovasc Dis. 2016:58:537-47.

3. Eckel RH, Kahn SE, Ferrannini E, Goldfine AB, Nathan DM, Schwartz MW, et al. Obesity and type 2 diabetes: What Can be unified and what needs to be individualized? Diabetes Care. 2011;34:1424-30.

4. Jung UJ, Choi MS. Obesity and its metabolic complications: the role of adipokines and the relationship between obesity, inflammation, insulin resistance, dyslipidemia and nonalcoholic fatty liver disease. Int J Mol Sci. 2014:15:6184-223

5. Esser N, Legrand-Poels S, Piette J, Scheen AJ, Paquot N. Inflammation as a link between obesity, metabolic syndrome and type 2 diabetes. Diabetes Res Clin Pract. 2014;105:141-50.

6. Guo H, Xia M, Zou T, Ling W, Zhong R, Zhang W. Cyanidin 3-glucoside attenuates obesity-associated insulin resistance and hepatic steatosis in high-fat diet-fed and db/db mice via the transcription factor FoxO1. J Nutr Biochem. 2012;23:349-60

7. Harzallah A, Hammami M, Kepczyńska MA, Hislop DC, Arch JRS, Cawthorne MA, et al. Comparison of potential preventive effects of pomegranate flower, peel and seed oil on insulin resistance and inflammation in high-fat and high-sucrose diet-induced obesity mice model. Arch Physiol Biochem. 2016;122:75-87

8. Ajiboye TO, Hussaini AA, Nafiu BY, Ibitoye OB. Aqueous seed extract of Hunteria umbellata (K. Schum.) Hallier f. (Apocynaceae) palliates hyperglycemia, insulin resistance, dyslipidemia, inflammation and oxidative stress in high-fructose diet-induced metabolic syndrome in rats. J. Ethnopharmacol. 2017;198:184-93.

9. Suwannaphet W, Meeprom A, Yibchok-Anun S, Adisakwattana S. Preventive effect of grape seed extract against high-fructose diet-induced insulin resistance and oxidative stress in rats. Food Chem Toxicol. 2010;48:1853-7.

10. Jung JY, Lim Y, Moon MS, Kim JY, Kwon O. Onion peel extracts ameliorate hyperglycemia and insulin resistance in high fat diet/streptozotocin-induced diabetic rats. Nutr Metab. 2011:8:18. https://nutritionandmetabolism. biomedcentral.com/articles/10.1186/1743-7075-8-18.

11. Saravanan G, Ponmurugan P, Deepa MA, Senthilkumar B. Anti-obesity action of gingerol: effect on lipid profile, insulin, leptin, amylase and lipase in male obese rats induced by a high-fat diet. J Sci Food Agric. 2014;94:2972-7.

12. Samuel VT. Fructose induced lipogenesis: from sugar to fat to insulin resistance. Trends Endocrinol Metab. 2011;22:60-5.

13. Lahlou M. The success of natural products in drug discovery. Pharmacol Pharm. 2013;04:17-31.

14. Li WL, Zheng HC, Bukuru J, De Kimpe N. Natural medicines used in the traditional Chinese medical system for therapy of diabetes mellitus. J Ethnopharmacol. 2004;92:1-21.

15. Buchholz T, Melzig MF. Medicinal plants traditionally used for treatment of obesity and diabetes mellitus - screening for pancreatic lipase and aamylase inhibition. Phyther. Res. 2016;30:260-6.

16. Hasani-Ranjbar S, Jouyandeh Z, Abdollahi M. A systematic review of antiobesity medicinal plants - an update. J Diabetes Metab Disord. 2013:28. https://pubmed.ncbi.nlm.nih.gov/23777875/

17. Wu LY, Juan CC, Hwang LS, Hsu YP, Ho PH, Ho LT. Green tea supplementation ameliorates insulin resistance and increases glucose transporter IV content in a fructose-fed rat model. Eur J Nutr. 2004:43: $116-24$

18. Carvajal-Zarrabal O, Nolasco-Hipolito C, Aguilar-Uscanga MG, Melo Santiesteban G, Hayward-Jones PM, Barradas-Dermitz DM. Effect of dietary intake of avocado oil and olive oil on biochemical markers of liver function in sucrose-fed rats. Biomed Res Int. 2014;2014.

19. Al-Muammar MN, Khan F. Obesity: The preventive role of the pomegranate (Punica granatum). Nutrition. 2012;28:595-604. https://www.sciencedirect. com/science/article/abs/pii/S0899900711004217.

20. Chen J, Mangelinckx S, Adams A, Wang ZT, Li WL, De Kimpe N. Natural flavonoids as potential herbal medication for the treatment of diabetes mellitus and its complications. Nat Prod Commun. 2015;10:187-200.

21. Amri Z, Zaouay F, Lazreg-Aref H, Soltana H, Mneri A, Mars M, et al. Phytochemical content, fatty acids composition and antioxidant potential of different pomegranate parts: comparison between edible and non edible varieties grown in Tunisia. Int J Biol Macromol. 2017:104:274-80.

22. Amri Z, Ghorbel A, Turki M, Akrout FM, Ayadi F, Elfeki A, et al. Effect of pomegranate extracts on brain antioxidant markers and cholinesterase activity in high fat-high fructose diet induced obesity in rat model. BMC Complement Altern Med. 2017:17:339.

23. Mekni M, Flamini G, Garrab M, Hmida RB, Cheraief I, Mastouri M, et al. Aroma volatile components, fatty acids and antibacterial activity of four Tunisian Punica granatum L. flower cultivars. Ind. Crops Prod. 2013;48:111-7.

24. Draper $\mathrm{HH}$, Hadley M. Malondialdehyde determination as index of lipid peroxidation. Methods Enzymol. 1990;186:421-31.

25. Levine RL, Garland D, Oliver CN, Amici A, Climent I, Lenz AG, et al. Determination of carbonyl content in Oxidatively modified proteins. Methods Enzymol. 1990;186:464-78.

26. Sun Y, Oberley LW, Li Y. A simple method for clinical assay of superoxide dismutase. Clin Chem. 1988;34(3):497-500.

27. Flohé L, Günzler WA. [12] Assays of Glutathione Peroxidase Methods Enzymol 1984;105:114-120

28. Baynes HW, Mideksa S, Ambachew S. The role of polyunsaturated fatty acids ( $n$-3 PUFAs) on the pancreatic $\beta$-cells and insulin action. Adipocyte Taylor \& Francis. 2018:7:81-7.

29. Wang X, Chan CB. N-3 polyunsaturated fatty acids and insulin secretion. J Endocrinol. 2015:224(3):R97-106. https://pubmed.ncbi.nlm.nih.gov/25486 966/. 
30. Watanabe Y, Tatsuno I. Omega-3 polyunsaturated fatty acids for cardiovascular diseases: present, past and future. Expert Rev Clin Pharmacol. 2017;10:865-73.

31. Bazinet RP, Layé S. Polyunsaturated fatty acids and their metabolites in brain function and disease. Nat Rev Neurosci. 2014;15(12):771-85. https://pubmed. ncbi.nlm.nih.gov/25387473/.

32. Wilczek MM, Olszewski R, Krupienicz A. Trans -Fatty acids and Cardiovascular Disease: Urgent Need for Legislation. Cardiol. 2017;138:254-8.

33. Matthews DR, Hosker JP, Rudenski AS, Naylor BA, Treacher DF, Turner RC. Homeostasis model assessment: insulin resistance and $\beta$-cell function from fasting plasma glucose and insulin concentrations in man. Diabetologia. 1985;28:412-9

34. Haffner SM, Miettinen H, Stern MP. The homeostasis model in the San Antonio heart study. Diabetes Care. 1997;20:1087-92.

35. Gerrits PM, Tsalikian E. Diabetes and fructose metabolism. Am J Clin Nutr. 1993,58(5 Suppl):796S-9S.

36. Lozano I, Van Der Werf R, Bietiger W, Seyfritz E, Peronet C, Pinget M, et al. High-fructose and high-fat diet-induced disorders in rats: impact on diabetes risk, hepatic and vascular complications. Nutr Metab. 2016;13:15. https://pubmed.ncbi.nlm.nih.gov/26918024/.

37. Roopchand DE, Carmody RN, Kuhn P, Moskal K, Rojas-Silva P, Turnbaugh PJ, et al. Dietary polyphenols promote growth of the gut bacterium akkermansia muciniphila and attenuate high-fat diet-induced metabolic syndrome. Diabetes. 2015;64:2847-58.

38. Den Besten G, Bleeker A, Gerding A, Van Eunen K, Havinga R, Van Dijk TH, et al. Short-chain fatty acids protect against high-fat diet-induced obesity via a pparg-dependent switch from lipogenesis to fat oxidation. Diabetes. 2015;64:2398-408

39. Ibrahim $M$, Bonfiglio $S$, Schlögl $M$, Vinales $K L$, Piaggi $P$, Venti $C$, et al. Energy Expenditure and Hormone Responses in Humans After Overeating HighFructose Corn Syrup versus Whole-Wheat Foods. Obesity. 2018;26(1):141-9.

40. Lin $\mathrm{Cl}$, Shen CF, Hsu TH, Lin SH. A high-fructose-high-coconut oil diet induces dysregulating expressions of hippocampal leptin and stearoyl-CoA desaturase, and spatial memory deficits in rats. Nutrients. 2017;9(6):619. https://pubmed.ncbi.nlm.nih.gov/28621759/.

41. Reddy SS, Ramatholisamma P, Karuna R, Saralakumari D. Preventive effect of Tinospora cordifolia against high-fructose diet-induced insulin resistance and oxidative stress in male Wistar rats. Food Chem Toxicol. 2009;47:2224-9.

42. Nasri R, Abdelhedi O, Jemil I, Daoued I, Hamden K, Kallel C, et al. Ameliorating effects of goby fish protein hydrolysates on high-fat-highfructose diet-induced hyperglycemia, oxidative stress and deterioration of kidney function in rats. Chem Biol Interact. 2015;242:71-80.

43. Vučić V, Grabež M, Trchounian A, Arsić A. Composition and potential health benefits of pomegranate: a review. Curr Pharm Des. 2019;25:1817-27.

44. Barrett A, Ndou T, Hughey CA, Straut C, Howell A, Dai Z, et al. Inhibition of a-amylase and glucoamylase by tannins extracted from cocoa, pomegranates, cranberries, and grapes. J Agric Food Chem. 2013;61:147786

45. Kam A, Li KM, Razmovski-Naumovski V, Nammi S, Shi J, Chan K, et al. A comparative study on the inhibitory effects of different parts and chemical constituents of pomegranate on a-amylase and a-glucosidase. Phyther Res. 2013:27:1614-20.

46. Adisakwattana S, Jiphimai P, Prutanopajai P, Chanathong B, Sapwarobol S, Ariyapitipan T. Evaluation of a-glucosidase, a-amylase and protein glycation inhibitory activities of edible plants. Int J Food Sci Nutr. 2010;61:295-305.

47. Les F, Arbonés-Mainar JM, Valero MS, López V. Pomegranate polyphenols and urolithin a inhibit a-glucosidase, dipeptidyl peptidase-4, lipase, triglyceride accumulation and adipogenesis related genes in 3T3-L1 adipocyte-like cells. J Ethnopharmacol. 2018:220:67-74.

48. Fabroni S, Ballistreri G, Amenta M, Romeo FV, Rapisarda P. Screening of the anthocyanin profile and in vitro pancreatic lipase inhibition by anthocyanincontaining extracts of fruits, vegetables, legumes and cereals. J. Sci. Food Agric. 2016;96:4713-23.

\section{Publisher's Note}

Springer Nature remains neutral with regard to jurisdictional claims in published maps and institutional affiliations.

\section{Submit your manuscript to a SpringerOpen ${ }^{\circ}$ journal and benefit from:}

- Convenient online submission

- Rigorous peer review

- Open access: articles freely available online

- High visibility within the field

- Retaining the copyright to your article

Submit your next manuscript at $\boldsymbol{\nabla}$ springeropen.com 